\title{
Effects of interface adhesive and resin cohesive strength on tensile strength of resin sand based on numerical analysis
}

\author{
Yun-dong Ji 1,2, Zi-han Liu ${ }^{1}$, Kai-dong Zheng ${ }^{1}$, Jia-bo Li ${ }^{3}$, Hai-xiao Hu ${ }^{2}$, Dong-feng Cao ${ }^{2}$ \\ 1. School of Materials Science and Engineering, Wuhan University of Technology, Wuhan 430070, China; \\ 2. State Key Laboratory of Advanced Technology for Materials Synthesis and Processing, Wuhan University of Technology, Wuhan 430070, \\ China; \\ 3. Zhangwu Country Lian Xin Foundry Silica Sand Co., Ltd., Fuxin 123200, China.
}

\begin{abstract}
A plane-strain unit-cell finite element model was proposed to study the effects of resin/sand interface adhesive and resin cohesive strength on the overall tensile strength of resin sand, as well as the fracture modes. The main micro-scale characteristics of the numerical model were extracted from the micrograph of resin sand specimens by three-dimensional X-ray microscopy (3D-XRM). The extended finite element method (XFEM) and cohesive behavior method were employed to explicitly describe the resin fracture and sand/resin interface debonding, separately. The corresponding experimental observation of micro-scale failure behavior based on the scanning electron microscopy (SEM) was presented for a comparison. The numerical results show that the initial failure of the model occurs at the sand/resin interface, followed by consequent resin failure. Dependent on the resin cohesive strength, the location of resin failure varies from the central zones to resin neck arc zones. A typical mixed mode fracture is observed, which is consistent with the corresponding micro-scale experimental observation. When the resin cohesive strength ranges between 8 and $12 \mathrm{MPa}$, the resin cracks occur at the central zone of resin bridges and propagate perpendicularly to the tensile direction until through cracks happen. At a higher range (between 12 and $16 \mathrm{MPa}$ ), interface cracks cross with resin cracks, bonding bridges of resin sand are broken. The interface adhesive strength has a more significant effect on the overall tensile strength of resin sand than the resin cohesive strength. The overall tensile strength of resin sand increases first then keeps stable with the increase of the resin cohesive strength. This work attempts to establish a numerical model which accurately describes the complicated mixed mode fracture of resin sand, which is beneficial to understand deeply the fracture mechanism of resin sand.
\end{abstract}

Key words: resin sand; microstructure; interface debonding; resin crack; fracture mode
CLC numbers: TG221 .2
Document code: A
Article ID: 1672-6421(2019)06-406-08

\begin{abstract}
$\mathrm{R}$ esin sand is one of the critical materials used in casting process ${ }^{[1]}$. It is well known that the mechanical strength of resin sand depends on the adhesive strength between the resin binder and sand grains, the cohesive strength of resin itself, and the resin distribution among sand grains. If the resin/sand interface stress level exceeds its adhesive strength, a debonding fracture occurs with the resin film peeling off the sand grains' surface. Conversely, if the stress level of resin is higher than its cohesive failure threshold,
\end{abstract}

\footnotetext{
*Dong-feng Cao

Male, Ph.D., Research Assistant. His research mainly focuses on the multiscale finite element methods, constitutive theories, microstructure optimization design.

E-mail: cao_dongf@whut.edu.cn
}

Received: 2019-06-03; Accepted: 2019-08-27 the resin bridge (or film) itself breaks off in the form of the cohesive fracture mechanism. Of course, a mixed fracture including the interface debonding and resin cohesive fracture can also be observed ${ }^{[2]}$. Zhang et al. ${ }^{[3]}$ experimentally studied the effect of resin/ sand interface adhesive strength on overall tensile strength $\left(\sigma_{\mathrm{b}}\right)$ of resin sand for varying conditions of environmental humidity and moisture content at raw sand surfaces. Interface layers were found to be the "weak link" deteriorating the overall tensile strength of resin sand. You et al. ${ }^{[4]}$ reported that the increased resin content not only resulted in higher thickness of resin films and contact surface area of bonding bridges, but also reduced the strength of coated sands. Moreover, an increase in resin content should be avoided in order to save production costs, exclude hazards to human 
health, and minimize environmental pollution ${ }^{[5-6]}$. Yang et al. ${ }^{[7]}$ proposed two mathematical models to predict the strength of resin sand. It is found that for the case of cohesive fracture, the overall tensile strength of resin sand is proportional to the resin content, and for the case of adhesion fracture, the overall tensile strength is proportional to the square of the resin content. The mathematical model performed by Zhu et al. ${ }^{[8]}$ strongly indicated that the overall tensile strength of resin sand was not affected by the particle size of raw sands, due to a competitive relationship between the reduction of resin film thickness (which reduces overall tensile strength of resin sand) and the increase in the number of bonding bridges. In particular, they reported that the specific surface areas of sand particles and bonding bridges' number rose with the particle size, which reduced the resin film thickness and the overall tensile strength. Meanwhile, an increase in the number of bonding bridges was found to be beneficial for the overall tensile strength of resin sand.

Massive effort has been carried out to study the impact of resin sand parameters, e.g. resin content, resin properties, the properties of resin/sand interface and the size of raw sand particles on the overall tensile strength of resin sand. However, the effects of spatial structure, bonding bridge curvature variation, and micro-fracture mechanisms received less attention ${ }^{[2-4,7-8]}$. Therefore, in this study, the microstructure of resin sand was observed via the high-solution three-dimensional X-ray microscopy (3D-XRM). Based on the observation result obtained, a plane-strain unit-cell finite element model of resin sand was constructed by the finite element method, which was implemented using the ABAQUS commercial software package. The proposed model was aimed to take into account the microstructural characteristics (such as the structure of resin-coated sand particles and pores between them, as well as interface curvature variation), capture the micro-fracture processes in detail, and identify their micro-fracture mechanisms under tensile loading conditions. Moreover, the proposed model was found applicable for assessing the effects of geometric structure and material properties (such as particle size distribution in raw sands, resin content, and resin cohesive properties) on the overall tensile strength of resin sand.

In our previous studies ${ }^{[9-10]}$, the calculation results strongly indicated that the overall tensile strength of resin sand could be improved by increasing the resin content and reducing the size of raw sand particles. The main drawback of the above studies is their failure to predict the effects of resin/sand interface adhesive and resin cohesive properties on tensile strength. Although the modification of resin/sand interface adhesive properties was reported to be quite effective for strength enhancement of resin sand in engineering practice ${ }^{[11]}$, their adjustment control and accurate measurements constitute a rather challenging task. In this study, effects of resin/sand interface adhesive and resin cohesive properties on the overall tensile strength were analyzed and correlated by changing parameters in the available literary data based on the proposed finite element model. In addition, tensile tests of resin sand samples were conducted, and their fracture modes were identified using scanning electron microscopy (SEM) for a comparison.

\section{Numerical unit-cell model}

\subsection{Numerical unit-cell model based on micrograph of resin sand}

In this work, the sample was prepared from ceramic foundry sands (70 meshes), phenolic resin (1.5 wt.\%) and isocyanate resin (1.5 wt.\%). Afterward, two-dimensional images of the sample at different angles were obtained by using 3D-XRM. Through this method, the actual spatial distribution of particles and geometric characteristics of bonding bridges were observed. Figure 1(a) shows the local two-dimensional image of the sample, with the relative density of each component being visualized by different colors: brighter circular zones are sand particles, lighter gray zones represent resin films, and black zones correspond to holes between particles. Moreover, the geometry information such as the thickness of resin film, the size of sand particles, and curvature radius of the resin neck were measured. In addition, the size of particles at the same horizontal plane also varies, due to different spatial accumulation of particles.

Microstructural characteristics, such as resin coating sand particles, particle size distribution of raw sands, and variation
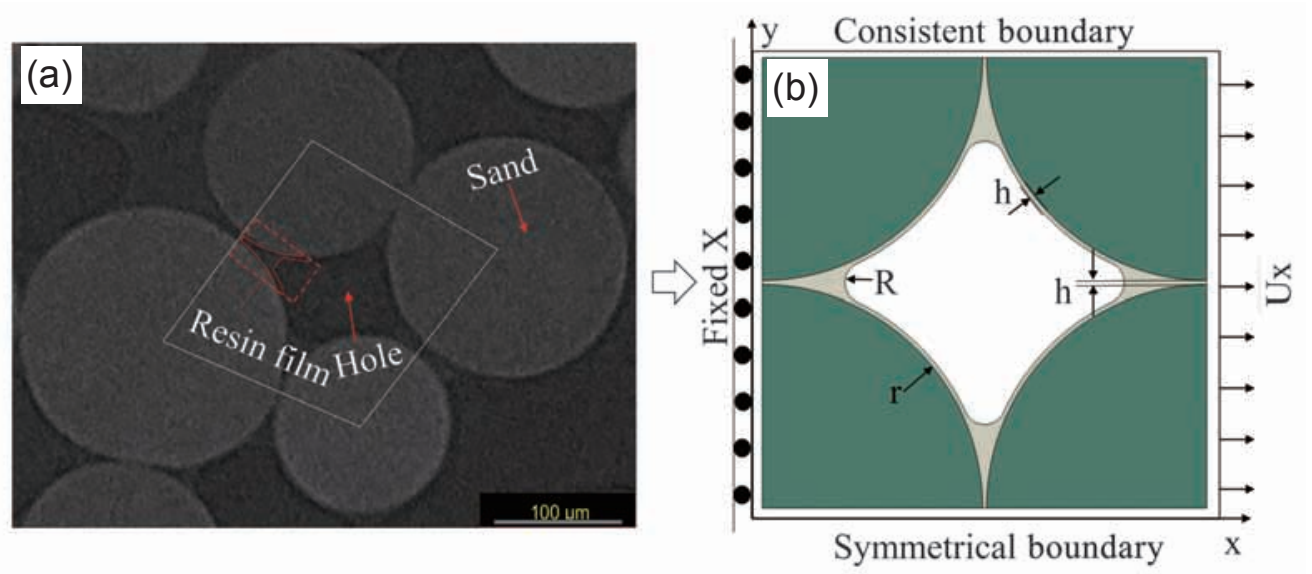

Fig. 1: Simplification process of two-dimensional resin sand model: (a) actual two-dimensional image of resin sand; (b) plane-strain unit-cell finite element model of resin sand 
of bonding bridge curvature, were assessed via micro computed tomography images. Then, according to the revealed resin sand microstructure, the unit-cell model of resin sand was constructed and run in ABAQUS. In addition, the plane-strain unit-cell finite element model of resin sand was simplified by neglecting the complex spatial structure of resin sand and the existence of water, impurities, and acidic substances on the surfaces of sand particles, as shown in Fig. 1(b).

The simplified model uses the following assumptions: (1) sand particles are treated as uniformly distributed solid spheres with a radius ( $r$ ) of $0.106 \mathrm{~mm}$ (70 meshes); (2) sand particles are coated with resin of $0.002 \mathrm{~mm}$ thickness $(h)$; (3) the resin distribution is uniform, without defects and other influencing factors; (4) the curvature radii $(R)$ of all resin neck arcs are assumed to be equal to $0.01 \mathrm{~mm}$.

The boundary conditions are as follows: (1) a fixed constraint in the X-direction is applied to the left surface; (2) a displacement load in the X-direction is applied to the right surface; (3) a symmetrical constraint in the Y-direction is imposed on the bottom surface; (4) a coupled constraint in the Y-direction is imposed on the upper surface.

The unit-cell model has been successfully used to simulate the micro- and macrostructural mechanical behavior of particlereinforced composites ${ }^{[10,12-14]}$. If the composite material under study is initially stress-free and its particles are evenly distributed within the matrix, each unit-cell meets the above boundary conditions ${ }^{[15-17]}$. Thus, the proposed unit-cell model of resin sand also complies with the above geometric conditions and boundary conditions. (a)
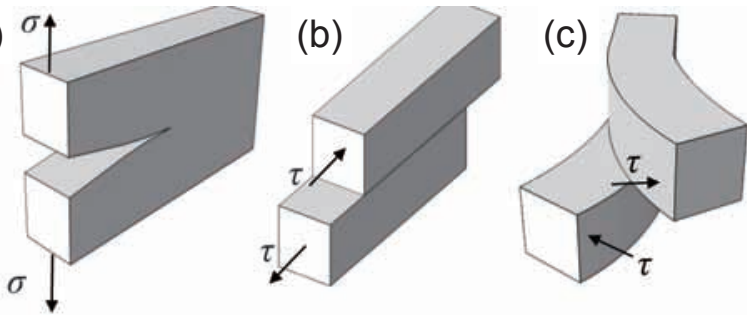

Fig. 2: Fracture modes: (a) Mode-l; (b) Mode-II; (c) Mode-III ( $\sigma$ is tensile stress; $\tau$ is shear stress)

\subsection{Impact of interface adhesive and resin cohesive strength on tensile properties of resin sand}

The cohesive behavior, which is controlled by interfacial bonding properties represented by the resin/sand interface adhesive strength, was considered in the further simulation of the interface failure behavior ${ }^{[18]}$. In general, there are three fracture modes, as shown in Fig. 2: (a) an open crack mode (Mode-I) controlled by the tensile stress, which direction is normal to the crack plane, (b) a slip mode (Mode-II) controlled by shear stress, which direction is normal to the crack front in the crack plane, and (c) a tear mode (Mode-III) controlled by shear stress, which is parallel to the crack front in the crack plane. Considering that Mode-III is absent in the plane-strain model, the interface strength assessed in this study corresponds to Mode-I and Mode-II. The failure behavior of the resin matrix is simulated by adopting the extended finite element method (XFEM) and the maximum principal stress failure criterion based on failure displacement ${ }^{[19-21]}$. The basic parameters used in the proposed model are listed in Table $1^{[9,22-28]}$.

Table 1: Material parameters of the model ${ }^{[9,22-28]}$

\begin{tabular}{|c|c|c|c|c|c|}
\hline Material & $E(\mathrm{GPa})$ & $v$ & $\sigma_{\mathrm{r}}(\mathrm{MPa})$ & $\delta(\mathrm{m})$ & \\
\hline Resin & 1.27 & 0.30 & 16 & 0.00002 & \\
\hline Sand & 50 & 0.13 & - & - & \\
\hline \multirow{2}{*}{ Cohesive behavior } & $\sigma_{\mathrm{l}}(\mathrm{MPa})$ & $\sigma_{\|}(\mathrm{MPa})$ & $G c_{l}\left(J \cdot m^{-2}\right)$ & $G c_{\|}\left(J \cdot m^{-2}\right)$ & $K_{\mathrm{nn}}=K_{\mathrm{ss}}\left(\mathrm{N} \cdot \mathrm{m}^{-1}\right)$ \\
\hline & 11.2 & 9.8 & 8 & 22 & $1 \times 10^{9}$ \\
\hline
\end{tabular}

Notes: $E$ is the Young modulus; $v$ is Poisson's ratio; $\sigma_{\mathrm{r}}$ is resin cohesive strength; $\delta$ is the displacement at failure of resin; $\sigma_{\mathrm{I}}$ and $\sigma_{\mathrm{II}}$ are Mode-I/II interface adhesive strength, respectively; $G \mathrm{c}_{\mathrm{I}}$ and $G \mathrm{c}_{\mathrm{II}}$ are Mode-I/II interface fracture energies, respectively; $K_{\mathrm{nn}}$ and $K_{\mathrm{ss}}$ are the normal and tangential resin/sand interface adhesive stiffness, respectively.

The experimental adjustment and accurate control of interfacial properties are quite problematic, so their effect on the overall tensile strength was numerically simulated via the proposed finite element model. The resin/sand interface adhesive strength of Mode-I/II $\left(\sigma_{\mathrm{I}}\right.$ and $\left.\sigma_{\mathrm{II}}\right)$ and resin cohesive strength $\left(\sigma_{\mathrm{r}}\right)$ were varied within an appropriate range according to the parameters listed in Table 1, and the respective 13 combinations obtained were abbreviated as Cases N1-N4, Q1-Q4, and S1-S5 in Table 2 .

These 13 cases were simulated by the proposed model using the above geometric and boundary conditions. In the postprocessing module, the resultant force $(F)$ acting along the tensile direction was derived by adding reaction forces of each node to the right surface of the unit-cell model. In addition, the thickness of the plane-strain model is defaulted to $1 \mathrm{~mm}$ and the tensile load of the model is calculated by dividing $\mathrm{F}$ by the side length of the model $(2 r+h)$. Finally, the peak value of the tensile load is defined as overall tensile strength of resin sand.

\subsection{Micro-fracture modes predicted by proposed model}

Figure 3 shows the maximum principal stress contour of the model during the tensile loading. Firstly, the local maximum principal stress is concentrated in the central zone of resin bridges, as shown in Fig. 3(a). Secondly, initial cracks appear at interfaces and then propagate along the interface direction during the 
Table 2: Applied combinations of interface adhesive and resin cohesive strength

\begin{tabular}{cccc} 
Case No. & $\sigma_{\text {I }}(\mathrm{MPa})$ & $\sigma_{\text {II }}(\mathrm{MPa})$ & $\sigma_{\mathrm{r}}(\mathrm{MPa})$ \\
\hline N1 & 7 & 9.8 & 16 \\
N2 & 9 & 9.8 & 16 \\
N3 & 11.2 & 9.8 & 16 \\
N4 & 13 & 9.8 & 16 \\
Q1 & 11.2 & 6.4 & 16 \\
Q2 & 11.2 & 7.9 & 16 \\
Q3 & 11.2 & 9.8 & 16 \\
Q4 & 11.2 & 13 & 16 \\
S1 & 11.2 & 9.8 & 8 \\
S2 & 11.2 & 9.8 & 10 \\
S3 & 11.2 & 9.8 & 12 \\
S4 & 11.2 & 9.8 & 14 \\
S5 & 11.2 & 9.8 & 16
\end{tabular}

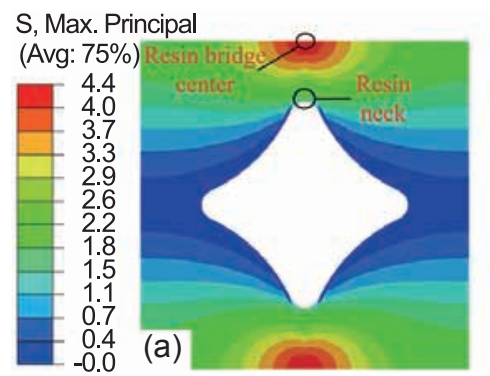

S, Max. Principal

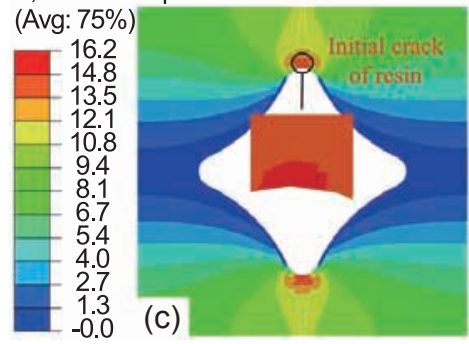

tensile load increase, as shown in Fig. 3(b). Due to the interface curvature, both tensile and shear stresses are generated at the interface paths but the former (local tensile) stress is dominant. However, when interface cracks propagate to resin neck arc zones, the fracture-controlling parameter is changed from local tensile stress to local shear stress. If the local shear stress at the interface does not exceed the threshold value of Mode-II crack, the maximum principal stress occurs at the resin neck arc. Cracks appear in the resin, as shown in Fig. 3(c), when the local stress in the resin neck reaches the $\sigma_{\mathrm{r}}$. A reduction in the loadbearing capacity of the model occurs due to cracking of resin and interface, and then the tensile stress reaches its peak value. Finally, the local shear stress at the interface becomes dominant, and resin crack propagation paths become irregular with an increase in the equivalent tensile displacement. Afterward, interface cracks cross with resin cracks, bonding bridges of resin sand are broken, leading to the complete fracture of bonding bridges and the loadbearing capacity loss of the model, as shown in Fig. 3(d). This tensile fracture mode of the model belongs to mixed fracture according to the above fracture process.

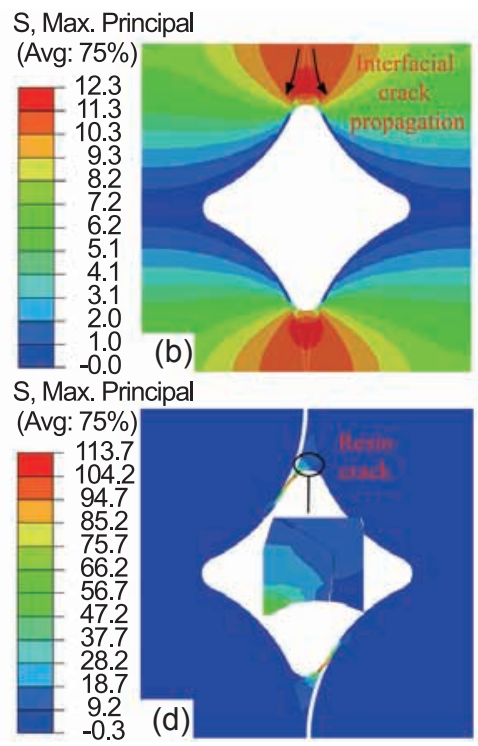

Fig. 3: Maximum principal stress contour during increasing equivalent tensile displacement: (a) initial loading stage; (b) interface crack propagation stage; (c) resin crack initiation stage; (d) resin crack propagation stage of the model

\section{Experimental set up, procedures and macro-scale fracture modes}

Four main raw materials were used in this work: ceramic foundry sands (70 meshes), phenolic resin (1.5 wt.\%), isocyanate resin $(1.5 \mathrm{wt} . \%)$, and trimethylamine. The mold size complied with GB/T2684-2009 standards ${ }^{[29]}$. Ceramic foundry sands, phenolic resin, and isocyanate resin were mechanically mixed in the sand mixer for 3 min successively. After that, the mixture was injected into the mold for $1 \mathrm{~min}$, while trimethylamine mixed with dry air was blown into the mold for $5 \mathrm{~s}$. Finally, samples were aged for $12 \mathrm{~h}$ after demolding.

Figure 4 shows two main fracture modes of samples under tensile loading conditions. A crack is initiated in the sample midsection, and the crack path was curvilinear. Figure 4(a) shows a typical tensile fracture mode of the sample. Due to a possible sliding of the sample during tensile loading, the crack path is inclined by 15 degrees to the horizontal plane, as shown in Fig. 4(b).

\section{Results and discussion}

\subsection{Micro-fracture SEM analysis}

The sample fractured surface was examined using SEM, as shown in Fig. $5^{[9-10]}$. A mixed fracture mode with interfacial fracture and resin matrix fracture is presented in Fig. 5(a), while Fig. 5(b) illustrates the resin film fracture with shear failure characteristics. These mixed fracture characteristics 

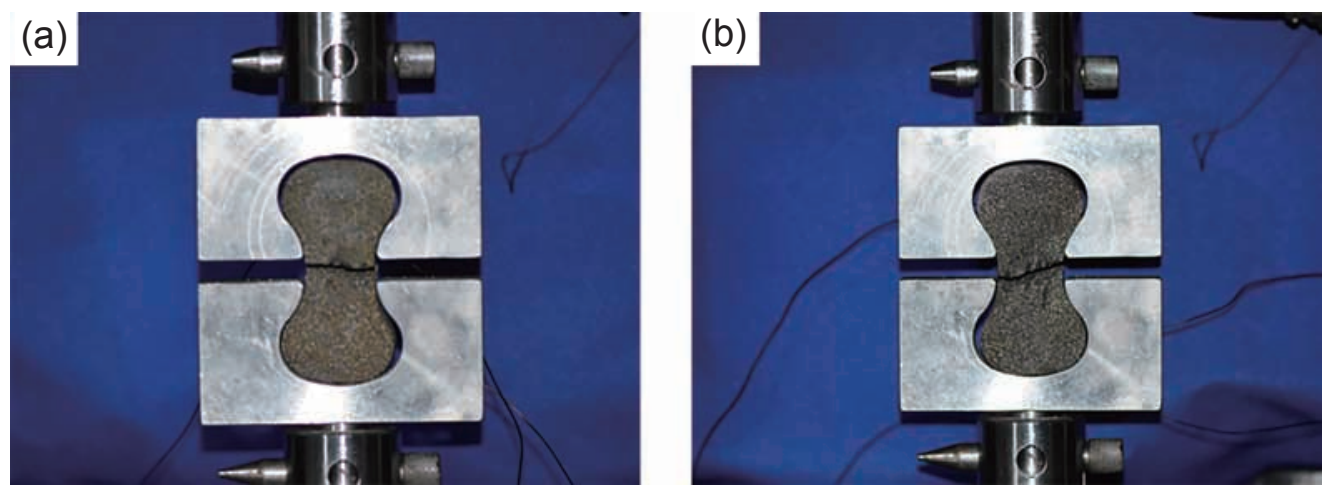

Fig. 4: Tensile fracture images of samples: (a) typical tensile fracture mode; (b) tensile fracture mode with an inclined crack path
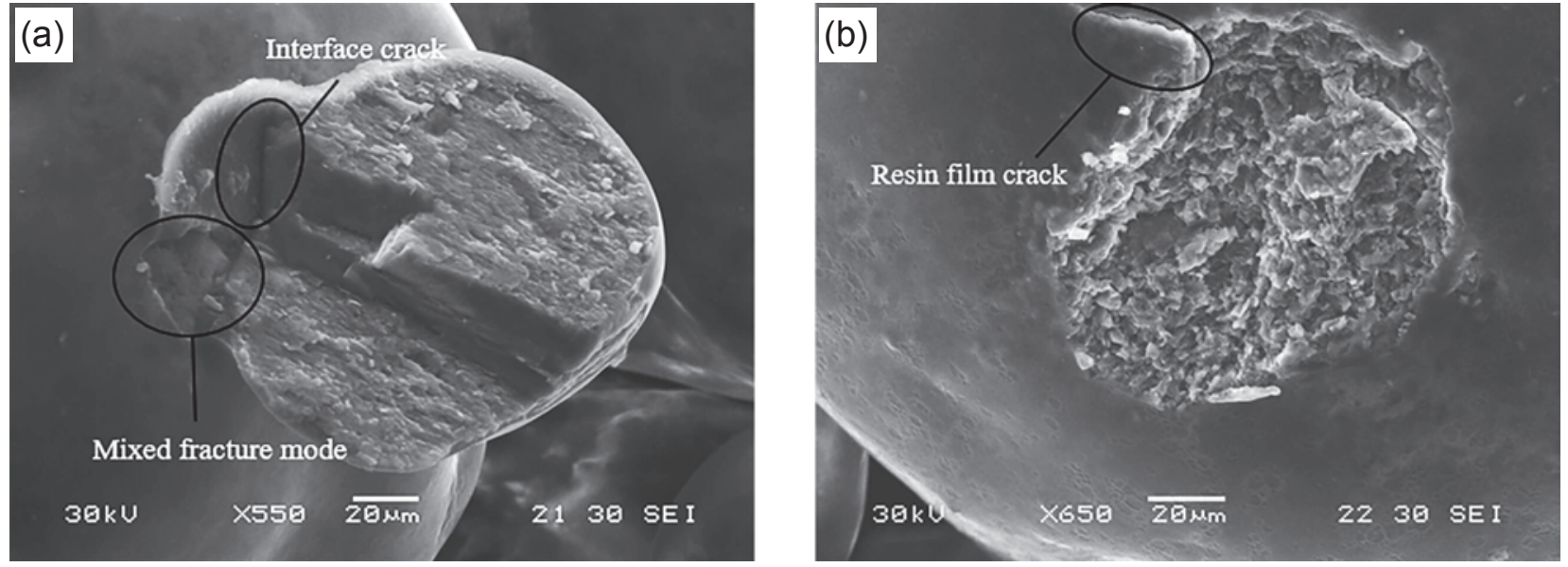

Fig. 5: Typical SEM images of tensile fracture surfaces of the sample: (a) SEM image of mixed fracture mode; (b) SEM image of resin film fracture ${ }^{[9-10]}$

were consistent with the predicted results by the proposed model based on cohesive behavior and XFEM.

\subsection{Effect of interface adhesive strength on tensile properties of resin sand}

Figure 6 shows the overall tensile strengths of resin sand $\left(\sigma_{\mathrm{b}}\right)$ for different Mode-I/II interface strength $\left(\sigma_{\mathrm{I}}\right.$ and $\left.\sigma_{\mathrm{II}}\right)$, respectively. In Fig. 6(a), the overall tensile strength grows from 2.9 to $5.1 \mathrm{MPa}$ (with an increment of $75.9 \%$ ) when parameter $\sigma_{\mathrm{I}}$ is increased from 7 to $13 \mathrm{MPa}$ (or by $86 \%$ ). In Fig. $6(\mathrm{~b})$, the effect of $\sigma_{\text {II }}$ on overall tensile strength is not obvious, since the overall tensile strength rises from 4.45 to 4.46 $\mathrm{MPa}$ with an increment of $0.22 \%$ (Cases Q1 and Q2). In Cases Q2-Q4, the overall tensile strength is close to $4.46 \mathrm{MPa}$.

Generally speaking, the interface adhesive strength has a prominent effect on the particle/matrix interface debonding behavior, and finally influences the tensile strength of particle reinforced composites ${ }^{[30]}$. The $\sigma_{\mathrm{I}}$ and $\sigma_{\text {II }}$ represent the bearing capabilities of normal tension and shear, separately. According to the numerical results in Fig. 6, the $\sigma_{\mathrm{I}}$ has more significant impact on the overall tensile strength of resin sand. In this pore structure, due to complicated geometry feature, the stresses
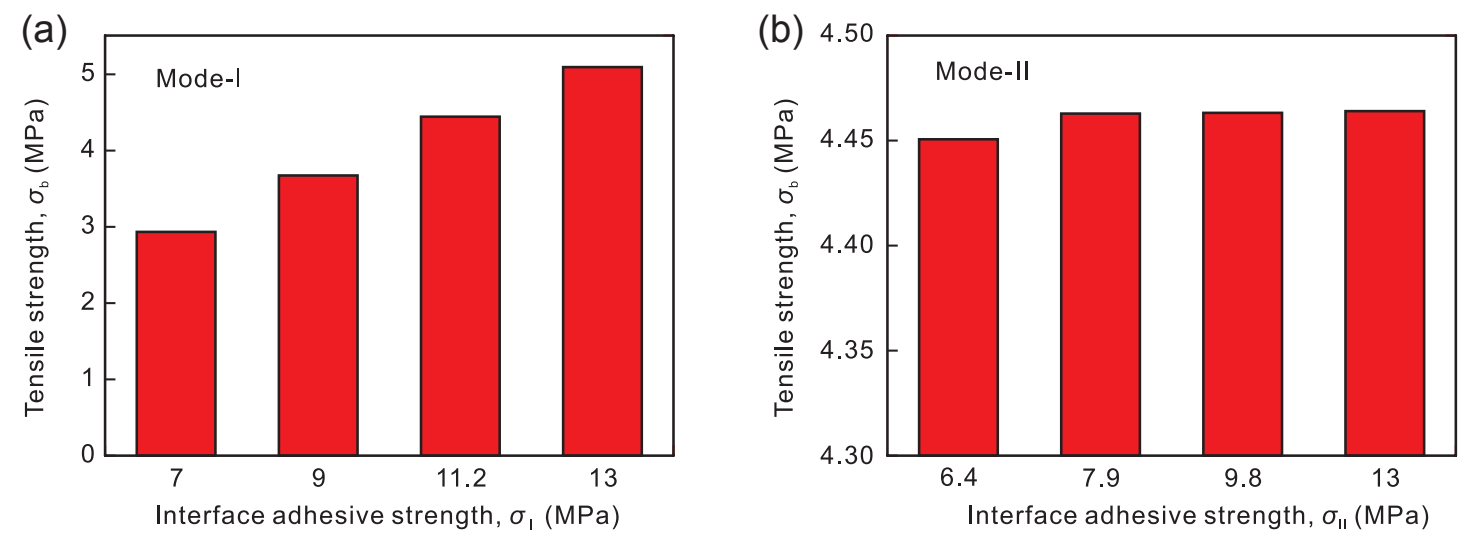

Fig. 6: Numerical calculation of models with different interface adhesive strengths: (a) Cases N1-N4 with various $\sigma_{\mid}$; (b) Cases Q1-Q4 with various $\sigma_{\|}$ 
in the resin and resin/sand interface are also complicated. The Mode-I interface tensile stress should play a more important role in the interface debonding. The increase of $\sigma_{\mathrm{I}}$ strengthens resistance capability for interface debonding due to Mode-I tensile stress. Consequently, the overall tensile strength of resin sand could be significantly influenced by the variation of $\sigma_{\text {I }}$ parameters.

\subsection{Effect of resin cohesive strength on tensile properties of resin sand}

Figure 7 shows the effect of resin cohesive strength $\left(\sigma_{\mathrm{r}}\right)$ on the overall tensile strength $\left(\sigma_{\mathrm{b}}\right)$ of resin sand. The overall tensile strength is improved gradually and then tends to be saturate with an increase in $\sigma_{\mathrm{r}}$. In particular, the overall tensile strength is increased by $22.6 \%$ (from 3.62 to $4.44 \mathrm{MPa}$ ) when $\sigma_{\mathrm{r}}$ increases from 8 to $12 \mathrm{MPa}$. With a further increase of $\sigma_{\mathrm{r}}$ to $16 \mathrm{MPa}$, the overall tensile strength grows only by $0.90 \%$ and reaches $4.48 \mathrm{MPa}$.

Figure 7 shows that the overall tensile strength of the resin sand increases gradually then keeps stable with the increase of the $\sigma_{\mathrm{r}}$, which is caused by the shift of fracture mode. In Cases S1 and S2 (with low $\sigma_{\mathrm{r}}$ ), cracks successively occur at the interfaces and resin bridges after the local maximum principal stress appears at the central zone of resin bridges. The Mode-I interface tensile stress should play a more important role in the interface debonding. Afterward, resin cracks propagate perpendicularly to the tensile direction, as shown in Fig. 8(a). The increase of $\sigma_{\mathrm{r}}$ strengthens resistance capability for resin cohesive failure at the central zone of resin bridges, which enhances the overall tensile strength of resin sand. This fracture mode is similar to the cohesive fracture, which was previously discussed ${ }^{[3]}$.

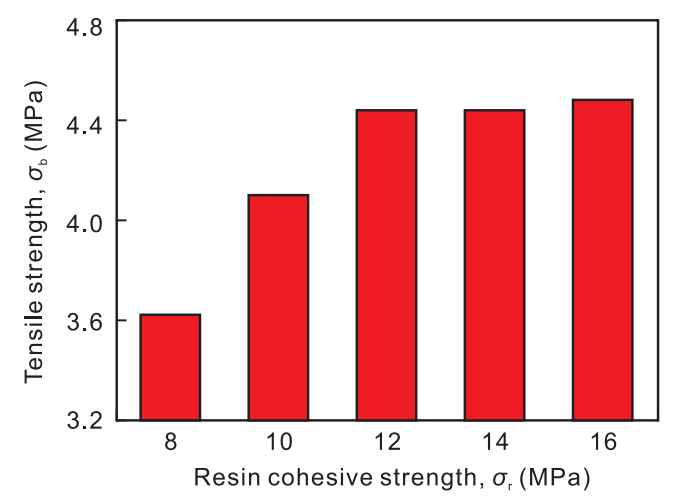

Fig. 7: Numeric calculation of model with different resin cohesive strength (Cases S1-S5)

Figure 8(b) shows the local failure mode of resin matrix in Case S5 (with high $\sigma_{\mathrm{r}}$ ), which has similar failure mode with Cases S3 and S4. The location of initial matrix failure is different from those of S1 and S2 (at the central zone of resin bridges). After the interface adhesive crack initiates and propagates, the maximum principal stress moves from the central zone of the resin bridge to the resin neck arc zone [see Fig. 3(a)-(c)]. When the principal stress reaches the $\sigma_{\mathrm{r}}$, the resin crack initiates and propagates. In addition, propagation paths of resin cracks are not normal to the tensile direction but have a

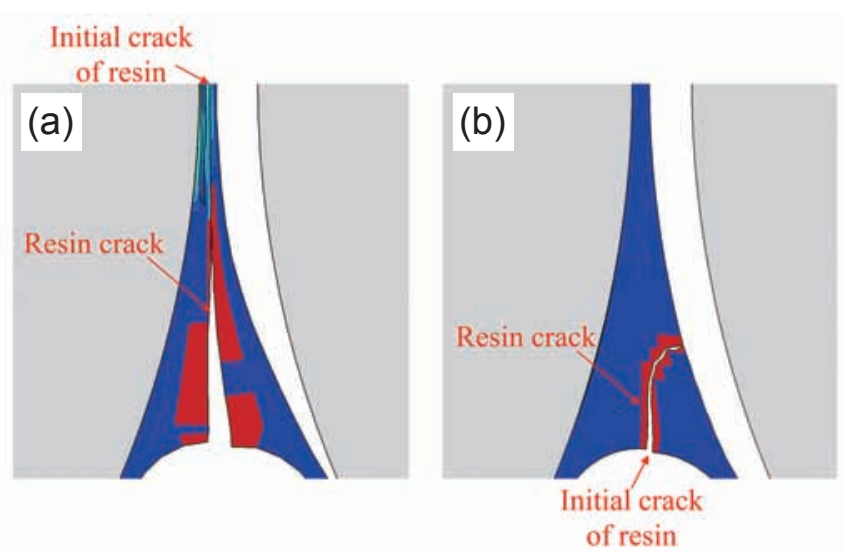

Fig. 8: Local failure mode of resin matrix for different combinations of $\sigma_{l}, \sigma_{\| \mid}$and $\sigma_{\mathrm{r}}$ : (a) Cases $\mathrm{S} 1$ and S2; (b) Cases S3-S5

certain inclination angle. This fracture mode corresponds to the mixed fracture, which was previously studied ${ }^{[3]}$.

The calculation results imply that variations of $\sigma_{\mathrm{I}}, \sigma_{\mathrm{II}}$ and $\sigma_{\mathrm{r}}$ would influence the overall tensile strength. In addition, fracture modes of the model change with an increase in $\sigma_{\mathrm{r}}$. According to the different fracture modes, the relevant two assumptions (i.e., ten-fold increase in $\sigma_{\mathrm{I}}$ and extremely low $\sigma_{\mathrm{II}}$ ) were made for Case S5.

The simulation result obtained implies that the initial crack also appears at the interfaces when $\sigma_{\mathrm{I}}$ is increased by ten times, as shown in Fig. 9(a). Meanwhile, the local maximum principal stress at the central zone of resin bridges exceeds $16 \mathrm{MPa}\left(\sigma_{\mathrm{r}}\right)$, leading to the resin cracking. Afterward, resin cracks propagate perpendicularly to the tensile direction by the fracture mode shown in Fig. 8(a). In addition, there would be no cracks at the resin neck arc vicinity when $\sigma_{\text {II }}$ was too low, as shown in Fig. 9(b). Meanwhile, interface cracks continue to propagate after interface cracks reached the resin neck arc zone, and this fracture mode is similar to the adhesion fracture.

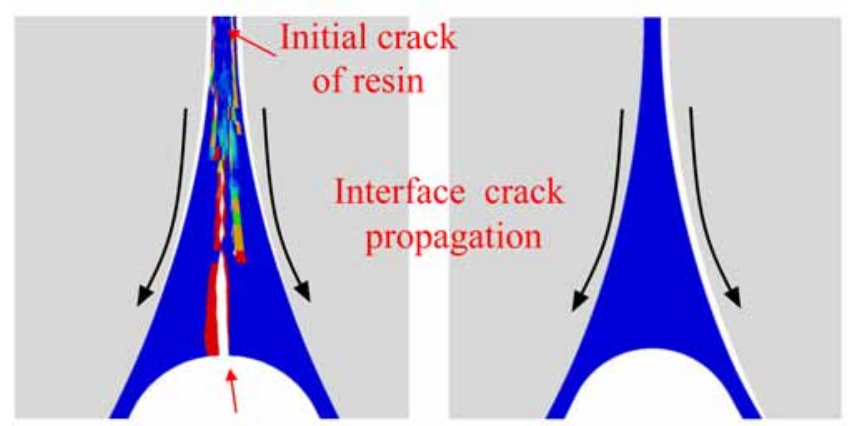

(a)

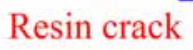

(b)

Fig. 9: Local failure modes of resin matrix for different assumptions; (a) Case S5 with ten-fold increase in $\sigma_{\mathrm{I}}$; (b) Case S5 with extremely low $\sigma_{\text {II }}$ and Case S5 without using XFEM

According to the above calculation results, some difference between the fracture modes predicted by the proposed model and via the conventional theory was observed, which could be attributed by the specific features of the microstructural 
model (such as the structure of resin-coated sand particles and pores between them, as well as interface curvature variation), as shown in Fig. 10. Due to the interface curvature, the interface of Zone A is mainly subjected to the tensile stress, while that of Zone B region to shear stress during the tensile load increase. Consequently, structural characteristic stress gradually changes from local tensile stress to shear stress in the interface crack propagation path. When $\sigma_{\mathrm{r}}$ is between 8 and $12 \mathrm{MPa}\left(\right.$ low $\sigma_{\mathrm{r}}$ ), the local maximum principal stress first occurs at the central zone of resin bridges, causing damages to the interfaces and resin successively, and then resin cracks propagate perpendicularly to the tensile direction. In addition, the interfacial local shear stress in the resin neck arc vicinity could reach the threshold value of the Mode II-type crack when $\sigma_{\text {II }}$ is extremely low. Then the interface cracks continue to propagate along the interface direction, while the resin neck arc zones remain undamaged. Thus, the propagation rate of interface cracks decreases when the interfacial shear stress in the resin neck arc vicinity is less than $\sigma_{\mathrm{II}}$. Then, the local maximum principal stress occurs in the resin neck zones, and the respective stress distribution is changed. Resin cracks appear at the resin neck arcs when local stress reaches $\sigma_{\mathrm{r}}$. Finally, resin cracks cross with interface cracks and induce the complete fracture of bonding bridges.

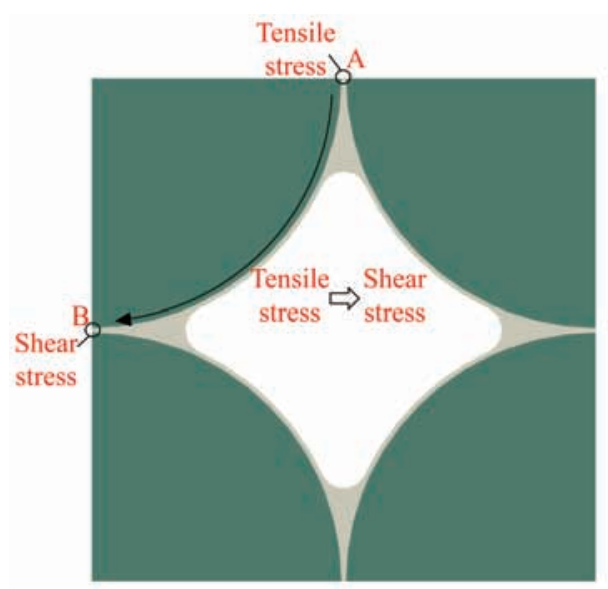

Fig. 10: Schematic map showing the shift from interfacial tensile stress-controlled fracture mode to shear stress-controlled

The above two assumptions concerning $\sigma_{\mathrm{I}}$ and $\sigma_{\text {II }}$ were made, according to the specific features of the microstructural model. Another assumption (i.e., the resin matrix would not fracture) was made for Case S5. The results obtained imply that initial cracks appear at the interfaces, as shown in Fig. 9(b), and their propagation rate drops when they reach the resin neck arc zones. Meanwhile, the local maximum principal stress at the resin neck arc zones is increased continuously, insofar as the resin matrix would not fracture, and the local shear stress at the interface plays the leading role. The calculation results strongly indicate that the overall tensile strength of the model reaches its peak value whether the resin shows fracture or nonfracture and only slightly exceeds that of Case S5. The reason is that the propagation of interface cracks is the main factor controlling the load-bearing capacity reduction, while the effect of $\sigma_{\mathrm{r}}(12-16 \mathrm{MPa})$ on the overall tensile strength is less noticeable. Besides, the propagation of the interface cracks and the fracture modes are related to the specific features of the microstructure model. Therefore, increasing the interface adhesive strength could noticeably improve the overall tensile strength and implies the mixed fracture mode.

\section{Conclusions}

In this work, the specific features of resin sand microstructure were identified with the aid of 3D-XRM and simulated by the proposed plane-strain unit-cell finite element model to study the effects of resin cohesive strength $\left(\sigma_{\mathrm{r}}\right)$, and resin/sand interface adhesive strength $\left(\sigma_{\mathrm{I}}\right.$ and $\left.\sigma_{\mathrm{II}}\right)$ on the overall tensile strength of resin sand, as well as the failure modes. The following conclusions were drawn:

(1) The overall tensile strength increases with $\sigma_{\mathrm{I}}$ and $\sigma_{\mathrm{II}}$, whereas variation of $\sigma_{\mathrm{I}}$ could more significantly improve tensile strength. In addition, the overall tensile strength is improved when $\sigma_{\mathrm{r}}$ increases to $12 \mathrm{MPa}$, and then keeps stable with a further increase of $\sigma_{\mathrm{r}}$ to $16 \mathrm{MPa}$.

(2) The cohesive behavior and XFEM effectively capture the fracture failure behavior of resin sand. When the model parameters are varied in certain ranges, cracks appear at interfaces and resin matrix successively. A typical mixed- mode fracture is presented and consistent with the corresponding micro-scale experimental observation.

(3) Due to the specific features of the microstructural model, the characteristic stress gradually shifts from the local tensile stress to the shear one during the interface crack propagation. When $\sigma_{\mathrm{r}}$ is between 8 and $12 \mathrm{MPa}$, resin cracks appear at the central zone of resin bridges and then propagate perpendicularly to the tensile direction by the fracture mode, which is similar to the cohesive one. In the range of $\sigma_{\mathrm{r}}$ between 12 and $16 \mathrm{MPa}$, resin cracks occur at the resin neck arcs and have irregular propagation paths, which correspond to mixed fracture mode.

\section{References}

[1] Ji Y D. Study on EPA modified phenolic resin and application for light-alloy casting. Huazhong University of Science and Technology, 2005. (In Chinese)

[2] Ding G, Zhang Q, Zhou Y. Strengthening of cold-setting resin sand by the additive method. Journal of Materials Processing Technology, 1997, 72(2): 239-242.

[3] Zhang Y N, Dong Y Y. The influence of weak boundary layer of the adhesive strength of binding bridge of resin-sand. Journal of Tianjin University, 1987, (4): 99-106. (In Chinese)

[4] You M, Huang N Y, Cao W L. The influence of resin content on the thermal properties of shell mold sand. Journal of Huazhong University of Science and Technology: Nature, 1989 (S2): 8994. (In Chinese)

[5] Evan D S, Dilpreet S B, Dean C W, et al. Epoxidized sucrose soyate - A novel green resin for crop straw based low density fiber boards. Industrial Crops and Products, 2017, 107. 
[6] Fox J T, Cannon F S, Brown N R, et al. Comparison of a new, green foundry binder with conventional foundry binders. International Journal of Adhesion and Adhesives, 2012, 34: 38-45.

[7] YANG J, LI O Q, Cheng J. Mathematical model of relationship between the resin addition levels and tensile strength of moulding sand in PEPSET Process. Foundry, 1997, (12): 1316. (In Chinese)

[8] Zhu Y L, Cai Z S. Analysis on the effect of raw sand size on the strength of resin-sand. Foundry, 1996, (12): 35-36. (In Chinese)

[9] Ji Y D, Zheng K D, Cao D F, et al. Numerical calculation of tensile failure behavior of resin sand composite material model based on extended finite element and cohesive behavior. Acta Materiae Compositae Sinica: 2019, (4): 1-9. (In Chinese)

[10] Ji Y D, Zheng K D, Cao D F, et al. Numerical study on tensile mechanical behavior of resin sand based on micro-scale unit cell model involving cohesive failure mechanism. Foundry, 2019, 68(04): 364-371. (In Chinese)

[11] Ye L, Ji Y D, Zhong F S, et al. Research on Surface Treatment of Silica Sand Used for Foundry with Carboxymethylcellulose Sodium. Hot Working Technology, 2017, 46(05): 97-100, 103. (In Chinese)

[12] Xue Z, Huang Y, Li M. Particle size effect in metallic materials: a study by the theory of mechanism-based strain gradient plasticity. Acta Materialia, 2002, 50(1): 149-160.

[13] Tvergaard V. Analysis of tensile properties for a whiskerreinforced metal-matrix composite. Acta Metallurgica et Materialia, 1990, 38(2): 185-194.

[14] Bao G, Hutchinson J W, McMeeKing R M. Particle reinforcement of ductile matrices against plastic flow and creep. Acta Metallurgica et Materialia, 1991, 39(8): 1871-1882.

[15] Bruzzi M S, Mchugh P E, O'Rourke F, et al. Micromechanical modelling of the static and cyclic loading of an Al 2124-SiC MMC. International Journal of Plasticity, 2001, 17(4): 565-599.

[16] Saraev D, Schmauder S. Finite element modelling of Al/SiCp metal matrix composites with particles aligned in stripes- a 2D3D comparison. International Journal of Plasticity, 2003, 19(6): 733-747.

[17] Cao D F, Duan Q F, Li S X, et al. Effects of thermal residual stresses and thermal-induced geometrically necessary dislocations on size-dependent strengthening of particlereinforced MMCs. Composite Structures, 2018, 200: 290-297.
[18] Michael A, Minnicino, Michael H. Santare. Modeling the progressive damage of the microdroplet test using contact surfaces with cohesive behavior. Composites Science and Technology, 2012, 72(16): 2024-2031.

[19] John Dolbow, Nicolas Moës, Ted, et al. An extended finite element method for modeling crack growth with frictional contact. Computer Methods in Applied Mechanics Belytschko, 2001, 190(51): 6825-6846.

[20] Nicolas Moës, Ted. Extended finite element method for cohesive crack growth. Engineering Fracture Mechanics Belytschko, 2002, 69(7): 813-833.

[21] Shi J X, David Chopp, Jim Lua, et al. Abaqus implementation of extended finite element method using a level set representation for three-dimensional fatigue crack growth and life predictions. Engineering Fracture Mechanics, 2010, 77(14): 2840-2863.

[22] Zhang D Y, Zhao Y, Wang G, et al. Main Methods of Improving Heat Resistance of Phenolic Resin. Heilongjiang Science, 2011, 2(05): 33-36. (In Chinese)

[23] Milad Zamanian, Mehrzad Mortezaei, Babak Salehnia, et al. Fracture toughness of epoxy polymer modified with nanosilica particles: Particle size effect. Engineering Fracture Mechanics, 2013, 97(1): 193-206.

[24] Ge D B, Wang S Z, Hu F Z. Study of phenolic resin and form modified by PEG and active polyethers. Fiber Reinforced Plastics/Composite, 2003, (6): 22-27. (In chinese).

[25] Wu R J. Application of modern analytical technology in polymer. Shanghai: Shanghai Scientific \& Technical Publishers, 1987. (In Chinese)

[26] Jin R G, Hua Y Q. Polymer physics: the third edition. Chemical Industry Press, 2007. (In Chinese)

[27] Huang Z X, Qin Y, Peng Y L. Thermosetting resin composites and their applications. Beijing: Chemical Industry Press, 2007. (In Chinese)

[28] Gu X L, et al. Subgrade and foundation, 2nd edition. Beijing: China Architecture \& Building Press, 1993. (In Chinese)

[29] GB/T 2684. Test methods for foundry sands and molding mixtures. National Standard of China, 2009. (In Chinese)

[30] Fu S Y, Feng X Q, Lauke B, et al. Effects of particle size, particle/ matrix interface adhesion and particle loading on mechanical properties of particulate - polymer composites. Composites Part B: Engineering, 2008, 39(6): 933-961.

The research work is supported by the Fundamental Research Funds for the Central Universities (WUT: 2018III066GX), Nature Science Foundation of Hubei Province (2017CFC809), China Postdoctoral Science Foundation (No. 2018M632933) and the Foreign Science and Technology Cooperation Project of Hubei Provenience (Grant No.2013BHE008). 